\section{Dr. Roimicher, et al, reply}

To the Editor:

We read the letter from Colavita, et $a l^{1}$, where the authors show by in vitro experiments that human osteoblast-like SV-40 T antigen immortalized (HOBIT) cells are productively infected by Zika virus (ZIKV). Their experiments with HOBIT cells infected with both Asian and African Zika strains show that both viruses are indeed able to produce a fair amount of virus $96 \mathrm{~h}$ postinfection. It is interesting that ZIKV could replicate in a HOBIT cell line and that we can find this kind of cell in normal articular tissue. A direct translation of their results to the case reported by us ${ }^{2}$ has to be put into perspective, however.

For instance, in the case presented by our group, a woman with rheumatoid arthritis (RA) had been in therapy-induced clinical remission for 4 months before being infected with ZIKV. The patient then presented with arthralgia and a clinical picture suggestive of ZIKV infection, which was confirmed by the presence of ZIKV genetic material in her knee synovial fluid by reverse transcriptase-PCR, even after the virus disappeared from her blood. Although the patient was in clinical remission for RA, her articular space could have been infiltrated by inflammatory cells such as macrophages, as well as activated lymphocytes that could be the major target cells for ZIKV replication. In ZIKV vertical transmission, human macrophages can be implicated because the virus has been detected in placental tissues of infected expectant mothers, specifically within the placental macrophages, known as Hofbauer cells, and intervillous histiocytes found on the maternal side ${ }^{3,4}$. Additionally, the neurotropism of ZIKV for glial cells and endothelial cells of fetal brains has also been identified ${ }^{4}$.

Nevertheless, the finding of direct infection of osteoblast cells by ZIKV reported by Colavita, et $a l^{1}$ offers an interesting alternative hypothesis for the arthritis caused by ZIKV infection. However, more work is needed to show what kind of cell cooperates with viral replication in the joint during the arthritis episodes induced by ZIKV infection. Immunohistochemistry analysis performed with specific monoclonal antibodies against ZIKV in cells found in a joint cavity of patients affected with clinical arthritis after ZIKV infection could help us to find the nature of cells infected by ZIKV.

LUIS ROIMICHER, MD, PhD, Professor of Rheumatology, Department of Rheumatology, Hospital Universitário Clementino Fraga Filho (CFF), Universidade Federal do Rio de Janeiro; ORLANDO C. FERREIRA JR., MD, PhD, Professor; MONICA B. ARRUDA, PhD, Professor; AMILCAR TANURI, MD, PhD, Laboratory of Molecular Virology, Centro de Ciências da Saúde, Universidade Federal do Rio de Janeiro, Rio de Janeiro, Brazil. Address correspondence to Dr. L. Roimicher, Department of Rheumatology-Internal Medicine, Hospital Universitário CFF, Universidade Federal do Rio de Janeiro, Rua Prof Rodolpho Paulo Rocco 255, 70 Andar, Rio de Janeiro, Brazil.

E-mail: mail-luizroimicher@gmail.com

\section{REFERENCES}

1. Colavita F, Musumeci G, Caglioti C. Human osteoblast-like cells are permissive for Zika virus replication. J Rheumatol 2018; 45:443-4.

2. Roimicher L, Ferreira OC Jr, Arruda MB, Tanuri A. Zika virus in the joint of a patient with rheumatoid arthritis. J Rheumatol 2017;44:535-6

3. Jurado KA, Simoni MK, Tang Z, Uraki R, Hwang J, Householder S, et al. Zika virus productively infects primary human placenta-specific macrophages. JCI Insight 2016;1(13)e88461.

4. Noronha Ld, Zanluca C, Azevedo ML, Luz KG, Santos CN. Zika virus damages the human placental barrier and presents marked fetal neurotropism. Mem Inst Oswaldo Cruz 2016;111:287-93.

J Rheumatol 2018;45:3; doi:10.3899/jrheum.171089 